\title{
Progress, prospect and challenges in glycerol purification process: A review
}

M.S. Ardi, M.K. Aroua, N. Awanis Hashim*

Department of Chemical Engineering, University of Malaya, 50603 Kuala Lumpur, Malaysia

A R T I C L E I N F O

Article history:

Received 8 July 2014

Received in revised form

16 October 2014

Accepted 25 October 2014

Available online 18 November 2014

Keywords:

Crude glycerol

Biodiesel

Purification

Membrane
A B S T R A C T

Glycerol surplus in recent decades due to global increase in biodiesel production has created a new form of challenge in terms of purification for crude glycerol. This review summarizes the progress of crude glycerol purification technologies using various techniques. Critical insights are given regarding the application of suitable techniques for crude glycerol purification which includes chemical pre-treatment, methanol removal, vacuum distillation, ion exchange, activated carbon and membrane separation technology. Extensive discussion is made in relation with stages and processes in the conventional, current and emerging glycerol purification technologies. Lastly, aspects concerning the challenges of glycerol utilization and purification are thoroughly discussed.

c 2014 Elsevier Ltd. All rights reserved.

Contents

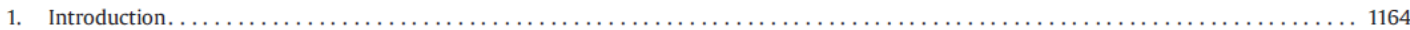

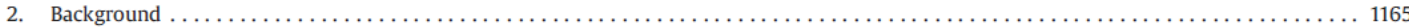

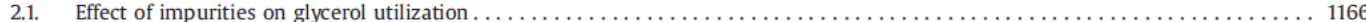

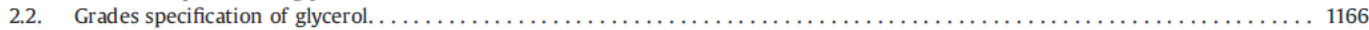

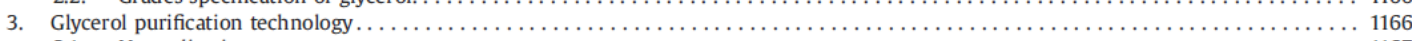

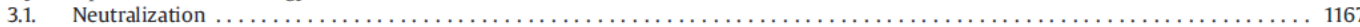

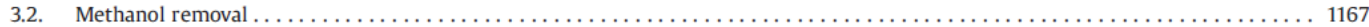

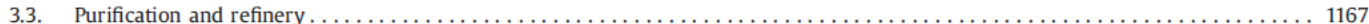

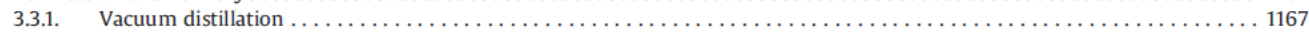

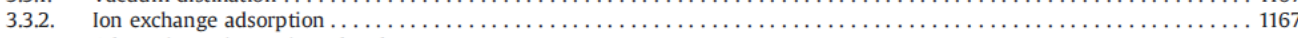

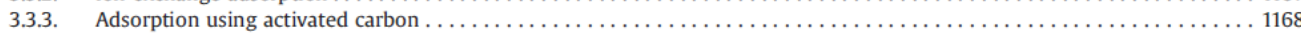

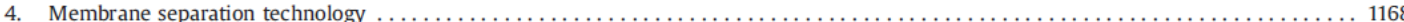

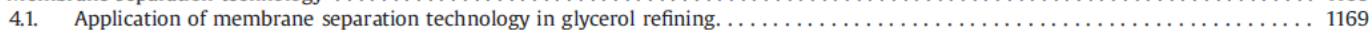

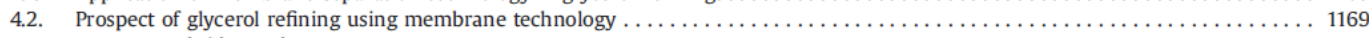

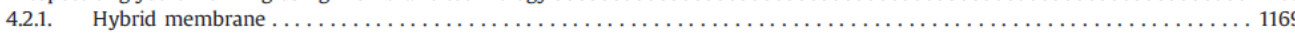

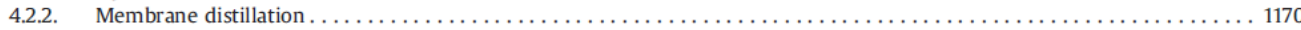

5. Challenges of crude glycerol utilization and purification. . . .

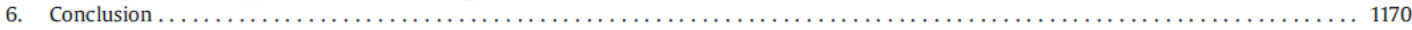

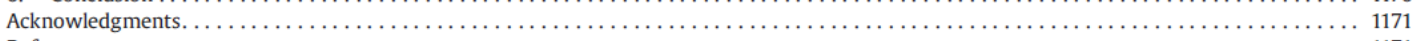

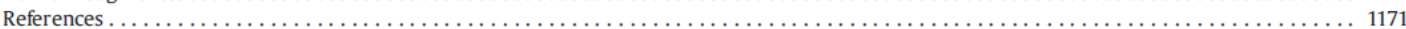

${ }^{*}$ Corresponding author. Tel.: +60379676892 .

E-mail address: awanis@um.edu.my (N.A. Hashim).

http://dx.doi.org/10.1016/j.rser.2014.10.091

$1364-0321 / \% 2014$ Elsevier Ltd. All rights reserved.

\section{Introduction}

As part of a recent renewable energy development interest, biodiesel has attracted numerous researchers worldwide as a viable alternative to rapidly decline fossil fuel consumption. Perhaps the wide interests are largely attributed to biodiesel 
attracting factors such as a renewable energy source, biodegradability, and low in hazardous air pollutant emissions $[1,2]$. Globally, crude glycerol is produced as the main by-products of the drastically increasing biodiesel production capacity. The rise in biodiesel production capacity has led to present situations whereby surplus of crude glycerol in the market contributes to cheaper glycerol prices. The crude glycerol produced which exceeds its demands further lowers its economic value [3]. Moreover, the crude glycerol produced contains a lot of contaminants which decreases its quality [4]. So the question now is - how exactly could we exploit crude glycerol or in other words, convert low value crude glycerol to value added chemicals. Application of the concept "from waste to worth" should be implemented for biodiesel production to further enhance the economic feasibility of the sustainable resources and exerting full capacity utilization of the main by-product, crude glycerol [5].

Crude glycerol is a valuable by-product of biodiesel production produced from animal, vegetable fats and oils such as palm oil as the feedstock. Approximately, the amount of crude glycerol produced is around $1 \mathrm{~kg}$ for each $10 \mathrm{~kg}$ of biodiesel produced [6-9]. Refining crude glycerol into a chemically pure substance increases its value for widespread uses and applications [4,10-12]. Glycerol is well known as a building block for a lot of chemicals reactions and applications $[13,14]$. There are already an extensive and huge amount of industrial application and day to day uses of glycerol in which they are very diverse in areas such as medical, pharmaceutical, food (sweetener in candies and cakes), toiletries (soaps and toothpastes), plasticizer, cosmetics, and lubricant in paper production, textile industry, surface coatings and printing inks. Glycerol is also widely used in production of several types of polymers as a side product of glycerol production [15-18]. Fig. 1 shows the distribution of glycerol consumption in different products and industries. Essentially, the importance of glycerol is apparent through its applications in key areas such as cosmetics, food and pharmaceutical products. Utilization of glycerol in these products requires one with high quality and purity.

Since the crude glycerol in its impure condition has a lesser value and usage compared to refined glycerol, series of purifications are usually required preferably prior to further processes [20]. Crude glycerol by-product of the transesterification process is classified as waste under Schedule S181 of the Environmental Regulations in Malaysia. It is stored in drums and disposed of in landfills [21]. Since the disposal of crude glycerol is costly, it is much advantageous to undergo purification process to generate income [9]. Current purification and refining technologies are already proven as an effective method to purify crude glycerol. However, due to the cost constraints and high energy requirement of the current method with exception to large scale plants, it is

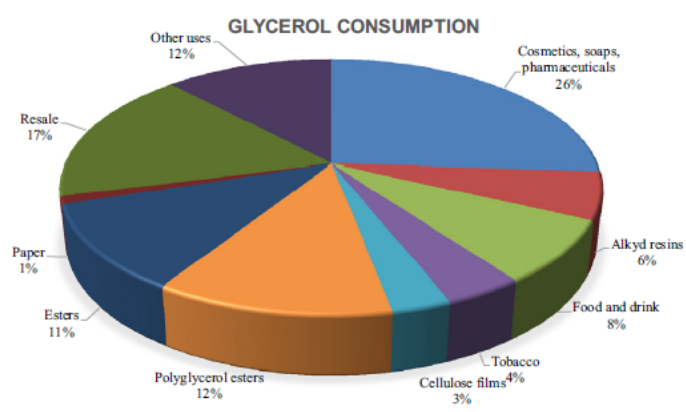

Fig. 1. Distribution of glycerol consumption in different products and industries [19]. deemed not feasible for small and medium plants as it has to undergo costly purification process [22-24].

Besides the advancement in the biodiesel production techniques, the recovery of high quality glycerol is identified to play a key role in biodiesel production cost reduction [25,26]. On-going development effort for cost reduction is visible through the improvement of better catalyst selection for biodiesel production, for example by employing heterogeneous catalyst which would produce purer glycerol with less contaminant [27]. Solid catalysts are not consumed or dissolved in the reaction and thus are easily separated from the products. As a result, impurities in the product that comes from the catalyst and the cost of final separation could be reduced [28].

In recent years, membrane technology has emerged as a promising technology to supplant the conventional glycerol purification method. Membrane technology offers green, environmental friendly and sustainable platforms with economic benefit. Aspects concerning environmental impact are reduced through process optimization, improvement in waste management and realization of cleaner processes to produce environmental friendly products.

Therefore, in this article we are interested to discuss more on the improvements of current and emerging glycerol refining techniques, particularly through the development of more efficient and cost effective glycerol purification processes. Challenges in purification and utilization of crude glycerol were also discussed as part of an effort to modify and further enhance the technology towards sustainable growth.

\section{Background}

Biodiesel is an alternative diesel fuel that contains a mixture of mono-alkyl esters of long chain fatty acid which was derived from vegetable oil or animal fat [29,30]. Direct usage of vegetable oils and animal fats as combustible fuel would not be suitable as it is high in kinematic viscosity and has low volatility. Therefore, in order to reduce the viscosity of the oils, vegetable oils and animal fats, they must be subjected to chemical reactions such as transesterification [31-33], direct/blends [34], micro-emulsion [35] and pyrolysis [36].

Transesterification is the most adopted technique for lowering viscosity and biodiesel production [37,38]. The viscosities of transesterified vegetable oil is closer to that of petrodiesel. Poor quality fuel injection and formation of deposits are the operational problem caused by high viscosity [39]. Fig. 2 presents the simplified transesterification process of triglyceride reaction to produce biodiesel and glycerol [40]. Transesterification of vegetable oils with alcohol can be carried out using either basic or acidic catalysts [41]. The reaction is reversible and thus to improve the reaction rate and yield, an excess amount of alcohol is used to shift the equilibrium to the product side [25,42-44].

Transesterification of triglycerides with alcohol along with catalysts such as sodium hydroxide produces methyl ester (biodiesel) phase and glycerol phase. Advantages of using the base catalyzed

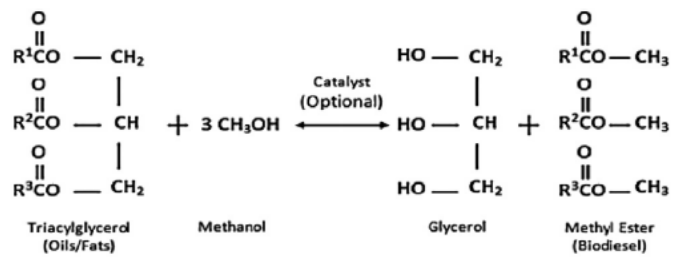

Fig. 2. Transesterification reaction of triglyceride [40]. 
transesterification process include high catalytic activity, low cost, favorable kinetics and modest operation condition which are some of the reasons why it is the most used technique for biodiesel production $[45,46]$. Base catalyst is also preferable due to relatively faster process and moderated reaction conditions [47]. It may be noted that plant oils with high free fatty acid contents suffer soap formation in the presence of homogeneous alkaline catalysts, leading to product loss and problems associated with product separation and purification [48]. On the other hand, while the use of acid catalysts would avoid soap formation, it is deemed not feasible due to issues such as corrosion, lower catalytic activity and higher temperatures requirement of above $100^{\circ} \mathrm{C}$ [49]. Table 1 shows the approximate amount of reactants and product amounts for transesterification reaction.

The usage of methanol as the primary alcohol in the biodiesel production is mainly attributed to its advantages as compared to other alcohols. Methanol is less expensive [51], high in reactivity using supercritical alcohol treatment [52,53], does not form azeotrope which would make the methanol recovery easier [54 and used for soap formation prevention during the biodiesel production [55]. Excess amount of methanol is frequently used as the reaction is a reversible process which can convert more fats and oil into biodiesel [55]. The impurities such as soap, methanol, water, salts, material organic non-glycerol (MONG) have more tendencies to concentrate in the glycerol phase during phase separation of biodiesel phase and glycerol phase [56,57].

\subsection{Effect of impurities on glycerol utilization}

Several studies have been conducted to investigate the characterization and effect of impurities on the glycerol for further process [58]. Impurities in crude glycerol has shown an inhibitory effect on high cell density production of phytase [59]. It was found that for initial concentration of $70 \mathrm{~g} / \mathrm{L}$ crude glycerol, certain impurities inhibited the cell growth. Soap and methanol have been found to negatively influence the algae production of DHA from crude glycerol [60] and the high salinity (Na or K) of crude glycerol can largely inhibit the microbial activity in the anaerobic digestion of crude glycerol [61]. Studies also show that saponified fatty acids (SFA) have inhibitory effect on photofermentative growth of Rhodopseudomonas palustris on crude glycerol to produce hydrogen [12]. The purification methods discussed in the

Table 1

Approximate reactant and product amounts for transesterification reaction [50].

\begin{tabular}{ll}
\hline Chemicals/feedstock/product & Amount (lbs) \\
\hline Oil/fat & 100 \\
Methanol & 22 \\
Catalyst & $0.3-0.5$ \\
Biodiesel & 100 \\
Glycerol & 11 \\
Excess methanol & 11 \\
\hline
\end{tabular}

study had found that pH adjustment and precipitation of SFA to be effective methods to reduce the SFA concentration.

Glycerol conversion of oxidation reaction over alumina supported platinum catalyst was found to decrease dramatically with the increase of matter organic non-glycerol - non-methanol (MONG-NM) [62]. In the study, a similar trend was observed in the presence of base and the absence of it. The main cause for decrease in the glycerol conversion that hinders oxygen dissolution was the presence of soaps and intensive foaming in which the catalyst surface was deactivated by strong adsorption of fatty acid derivatives. In addition, for pharmaceutical and food applications, heavy metals in crude glycerol might be harmful and present safety concerns [60]. Methanol is a toxic chemical which could cause damaging and harmful effects on health and environment [55]. Due to various applications of glycerol especially in pharmaceuticals and food related sectors, glycerol produced must meet certain requirements and standards.

\subsection{Grades specification of glycerol}

The goal of the purification process is to enhance usability of glycerol by removing unwanted impurities according to different sets of standard and purposes. Refined glycerol found in the market comprises of three basic types which is based on their purity and potential end use namely, technical grade, United States Pharmocopeia (USP) and Food Chemical Codex (FCC) [3]. Technical grade is mainly used as a chemical products building block and not for food and medicine. United States Pharmacopeia (USP) glycerol which is derived from animal fat or vegetable oil is suitable for both pharmaceutical and food products while the Food Chemicals Codex (FCC) glycerin which is mainly derived from vegetable oil sources is appropriate for use in food. The grades of glycerol according to purities are listed in Table 2.

\section{Glycerol purification technology}

Crude glycerol produced from various feedstock would have different compositions, therefore the purification process is also necessarily different. The consideration for initial purification or final purification of glycerol utilization pathway is largely dependent on the usage of glycerol and the effects of the impurities on the process, which would be part of cost control and profit measures. In cases where the glycerol utilization is mostly for technical applications, the presence of some contaminants is

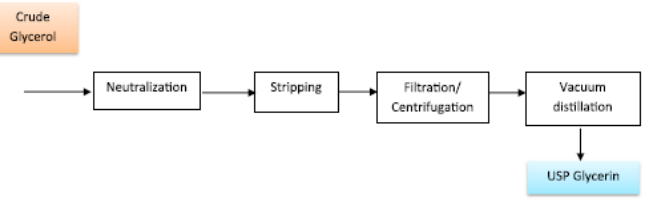

Fig. 3. General stages in the glycerol purification process [56].

Table 2

General specification of glycerol based on grades $[3,15,62]$.

\begin{tabular}{lll}
\hline Types of glycerol & Glycerol content $(\%)$ & Sources/applications \\
\hline Crude glycerol & $70-90 \%$ & By-product of biodiesel production \\
Technical grade & $99.5 \%$ Technical grade (not certified, mostly $>96.0 \%)$ & Suitable for industrial application \\
United States Pharmacopeia (USP) & $99.5 \%$ USP (tallow-based) & Used in cosmetic, pharmaceutical and food \\
& $99.5 \%$ USP (vegetable-based) & \\
Food Chemical Codex (FCC) & $99.7 \%$ USP/FCC-Kosher & \\
\hline
\end{tabular}


acceptable but for most cases, application of glycerol in food and pharmaceutical sectors would require high purity glycerol. The technology developed for purification of glycerol - derived from biodiesel - is mainly adapted from existing soap making industry, that utilizes vacuum distillation $[63,64]$ and other treatments such as ion exchange [65-67] and activated carbon [68,69]. As a rule of thumb, a general purification of glycerol processes comprise of three steps [70]. The first step involves the removal of non-glycerol substances which can be achieved through precipitations during neutralization, whereby free fatty acids and some salts are removed. The next step is to concentrate the solution by evaporation in which alcohol is removed from the glycerol stream. The final step is the purification and refining step which can be achieved to the desired degree with a combination of these methods which are vacuum distillation, ion exchange, membrane separation and adsorption. Fig. 3 shows the general stages in glycerol purification.

\subsection{Neutralization}

Neutralization is the most common method as pre-treatment of crude glycerol purification processes involving a chemical reaction using a strong acid to remove catalyst and soaps. The reaction of acid with soap would produce free fatty acids and its reaction with base catalyst would give salt and water. The insoluble free fatty acids and some salts will rise to the top and can be easily skimmed off. The first step through the acidification process usually separates the crude glycerol into three layers of free fatty acids at the top, glycerol rich layer at the middle and inorganic salts at the bottom.

Kongjao et al. reported that repeated acidification for crude glycerol derived from waste used-oil methyl ester plant using $\mathrm{H}_{2} \mathrm{SO}_{4}$ to the desired $\mathrm{pH}$ of 1-6 increases the yield of glycerol-rich layer obtained [6]. High purity of glycerol was obtained at $\mathrm{pH} 1$, 93.34\% with relatively low contaminant levels after a series of chemical, neutralization with $12.5 \mathrm{M} \mathrm{NaOH}$ and physical treatment. Attempt by Ooi et al. to recover crude glycerol from glycero residue using $6 \%(\mathrm{v} / \mathrm{v}) \mathrm{H}_{2} \mathrm{SO}_{4}$ reports that at a low $\mathrm{pH}$, the recovered glycerol was increased, ash content was reduced but the matter organic non-glycerol (MONG) was slightly increased 64]. Tianfeng et al. proposed acidification of crude glycero obtained from waste cooking oil biodiesel production using $5.85 \% \mathrm{H}_{3} \mathrm{PO}_{4}$ and founded an increase in volume of glycerol-rich layer content from $40 \%$ to $70 \%$ at pH 5 or 6 [71]. Acceptable yield of semi-product glycerol of $81.2 \%$ was obtained after acidification at optimum temperature of $70^{\circ} \mathrm{C}$ and reaction time of $60 \mathrm{~min}$. Further treatment using vacuum distillation resulted in acceptable glycerol purity of $98.10 \%$. Hajek et al. proposed acidification of glycerol phase from transesterification of vegetable oil through addition of strong acid which converts impurities such as soaps to higher fatty acids [72]. Meanwhile, Javani et al. proposed that crude glycerol to go through saponification and produce potassium phosphate as by-product using repeated acidification [73]. This is part of their proposed strategy to economize biodiesel production while producing high quality free fatty acids and glycerol due to the high price of the products.

\subsection{Methanol removal}

The second step in the general purification process is the removal of methanol. In the transesterification process, excess methanol is used in most biodiesel production to get high yield of biodiesel. The excess methanol is distributed between the methyl ester and crude glycerol phase [74]. The concern of major health safety and environmental issue for residual methanol in both biodiesel and glycerol is due to the toxicity of methanol in which the emission of excess methanol can have serious effects to the environment and public health. Therefore, the excess amount of methanol needs to be removed from the glycerol phase and recycled back [55]. Methanol and water can be easily removed by vacuum evaporation. For methanol removal, crude glycerol was treated under vacuum conditions using a rotary evaporator at $50-90{ }^{\circ} \mathrm{C}$ for more than $2 \mathrm{~h} \mathrm{[75]}$. It is a common practice in the industry to remove alcohol from both glycerol and biodiesel phase to utilize either an evaporator or a flash unit [76]. However, due to glycerol temperature susceptibility which can result in glycerol decomposition, using falling film evaporators was found to be more suitable and advantageous due to the fact that it keeps shorter contact time. The purity of crude glycerol after methanol removal will be approximately around $85 \%$.

\subsection{Purification and refinery}

\subsubsection{Vacuum distillation}

Distillation is generally not applicable to feed streams which are sensitive to thermal degradation or polymerize at elevated temperatures. Operation of column under vacuum can help to reduce or eliminate this problem [77]. Polymerization of glycerol into polyglycerol would occur at temperature higher than $200{ }^{\circ} \mathrm{C}$, dehydration in slightly acidic conditions at temperature above $160{ }^{\circ} \mathrm{C}$, and oxidation of glycerol into glycerose, glyceraldehydes and di-hydroxylacetone [78]. In order to prevent degeneration of glycerol, purification has to be done in vacuum where the $\mathrm{pH}$, temperature and pressure must be controlled. This is primarily due to an adverse reaction that causes degradation of glycerol. These are the reasons why vacuum distillation is the most common method for glycerol purification $[75,79,80]$.

Yong et al. reported that the purification of crude glycerol by simple distillation at $120-126{ }^{\circ} \mathrm{C}$ would yield around $141 \mathrm{~g}$ glycerol $/ \mathrm{kg}$ of glycerol residue ( $\sim 14 \%$ yield) at an acceptable purity of $96.6 \%$ glycerol [64]. The distilled glycerol product is of pH 3.5 consisted of $0.03 \%$ ash, $1 \%$ water, $2.4 \%$ matter organic non-glycerol (MONG). It was found that the optimum $\mathrm{pH}$ for the distillation was less than 5 in which foaming was avoided. The distillation process is a well-established technology [39], applicable for small to largescale continuous operation, low cost of chemicals as well as process adaptability that suits varying qualities of crude and finish products [81]. However, it should be noted that the distillation of crude glycerol is an energy intensive process [39]. High-energy input requirement for vaporization and creates thermal decomposition due to high specific heat capacity of glycerol which makes the process costly [52].

\subsubsection{Ion exchange adsorption}

Several types of impurities such as fatty acid, inorganic salt and free ion impurities can be removed from crude glycerol by using ion exclusion purification techniques [66,82]. The first early commercial application of ion exchange unit for crude glycerol (soap lye) purification at Lever Brothers Company plant in Los Angeles, California is of a four-stage unit consisted of three pairs of cation-anion exchangers and a mixed-bed. The plant which started its operation in May 1951 has the purification capacity of $26,600 \mathrm{lbs}$ of crude glycerol per day. The feed stream, crude glycerol produced from soap lye normally contains $82 \%$ glycerol, $10 \%$ ash ( $6 \%$ of the ash is the $\mathrm{NaCl}$ ), moisture and other impurities. The final product produced is exceptionally high in quality which compares favorably with high-grade distilled glycerol. The 95-99\% glycerol content confirms the pilot plant findings that indicate the process holds a number of advantages over the conventional distillation method. Among the advantages are low capital investment and exceptionally high product quality [82] which makes it 
more suitable for smaller capacity plant [39]. It is also applicable to a number of glycerol feed solutions such as separation of ionic impurities directly from spent soap lye solution (contains $5-10 \%$ glycerol, $12-15 \%$ salt) or solutions obtained from synthetic glycerol processes (contains 7-8\% glycerol, 12-15\% salt) [66]. However, later it was found that the technique results in poor separation due to fewer exchange sites per unit volume (smaller exclusion factor). Improvement based on this technique for crude glycerol from biodiesel production is introduced where gel type acidic ion exchange resin beads were used to eliminate several types of salt such as fatty acid salts (5-50\%), inorganic salts (1-5\%) and free ions impurities [83].

Ismail et al. utilized resin type AmberliteIRN-78 and Amberlite200 to purify crude glycerol and the effectiveness of resin was investigated [84]. Column techniques were performed in the experiments, where the ion-exchange resins were placed in vertical column forming a bed. High Performance Liquid Chromatography (HPLC) was used to analyze the glycerol samples obtained after the purification. A single glycerol peak with a very smooth baseline that matched to the pure glycerol profile was shown on the chromatograms for the purified samples. These results indicated that the application of the ion-exchange resin method is able to remove the impurities such as inorganic salts and free ions. However, for crude glycerol purification to be viable, issues concerning the ion exchange method such as fouling by fatty acids, oils and soaps, regeneration of the beds and large quantities of wastewater produced needs further improvement [39].

\subsubsection{Adsorption using activated carbon}

Adsorption with activated carbon is mainly used as the finishing step to further refine the purified glycerol; reduce the color, as well as reducing some fatty acids and other components. Manosak et al. used a commercial activated carbon in a sequential-refining stages process where it was found that increasing the dose of activated carbon had a significant effect on the color removal of the refined crude glycerol [11]. They found that a clear color (99.7\%) reduction was achieved with higher dose of activated carbon at $200 \mathrm{~g} / \mathrm{L}$ glycerol. During this stage, some of the fatty acids, such as lauric acid and myristic acid were eliminated. Although other fatty acids, such as palmitic acid, oleic acid and steric acid, as well as and some unknown compounds were still present, the refined glycerol were all in the acceptable range of values of the BS 2621:1979.
Hunsom et al. in their work prepared a set of 15 activated carbon derived from sewage sludge of waste water plant [4]. After the two steps of the sludge derived activated carbon preparation, pyrolysis and activation using different chemicals, $\mathrm{KOH}, \mathrm{K}_{2} \mathrm{CO}_{3}$ and $\mathrm{H}_{3} \mathrm{PO}_{4}$, adsorptive purification was carried out to pre-treated crude glycerol. It was found that from all prepared sludge derived activated carbon, one in particular namely $\mathrm{KOH}-800 \mathrm{AC}$ exhibite the highest efficiency to adsorb impurities in pre-treated crude glycerol. Based on the adsorptive result comparison between commercial activated carbon and $\mathrm{KOH}-800 \mathrm{AC}$, the latter showed improved glycerol purity. Adsorptive purification using $\mathrm{KOH}-$ $800 \mathrm{AC}$ is 1.06 -fold higher than that obtained with the commercial activated carbon (84.1 wt\%).

\section{Membrane separation technology}

The membrane separation process is a very simple process. The driving force of the most isothermal membrane separation process involves the difference in concentration or electrical potential and hydrostatic pressure. Employment of different types of membranes, microfiltration (MF), ultrafiltration (UF), nanofiltration (NF), reverse osmosis (RO) and electrodialysis (ED) has been reported for various applications [85-91]. Different types of membrane are employed for different types of separation process, for example microfiltration involves in separation of particles, whereas separation of macromolecules utilizes ultrafiltration and reverse osmosis is capable of separating ionic components [89]. A membrane is a barrier, which separates two phases and restricts transport of various chemical species in a selective manner [92]. Either one of the permeate and retentate be the end-use products in a membrane-based separation process [93]. Liquid separation of various chemicals-based product using membrane based process are usually employed for concentration and purification operations, water and waste water treatments. The wide applications of membranes include employment of the mentioned process in

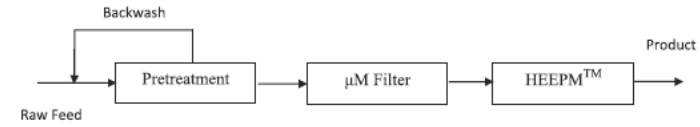

Fig. 4. EET corporation glycerol purification using membrane separation technology [56].

Table 3

List of membranes and filters used for glycerol purification.

\begin{tabular}{|c|c|c|c|}
\hline Membranes/filters & Material/types & Process & References \\
\hline Ultram SS316L/TiO 2 (USA) & Ceramic & & \\
\hline нІтк, Germany & Ceramic & & \\
\hline NF30 PP100 (Nadir NF Membranes, Celgard, Germany) & Polypropylene, organic & Nanofiltration & \\
\hline PVDF GE osmonic & $\begin{array}{l}\text { Polyvinylidene, } \\
\text { Organic } \\
\text { Flat sheet }\end{array}$ & Ultrafiltration & Mah et al., [96] \\
\hline \multirow[t]{2}{*}{ PES and PVDF } & Organic & Ultrafiltration & Indok et al., [98] \\
\hline & Organic & Nanofiltration & Traving [99] \\
\hline DL (NF) GE osmonics & Organic & Nanofiltration & Muralidhara [100] \\
\hline SG (RO) GE osmonics & & Reverse osmosis & \\
\hline \multicolumn{4}{|l|}{ SR2 (NF) Koch } \\
\hline \multicolumn{4}{|l|}{ SR3 (NF) Koch } \\
\hline TRS83 (NF) TRISPE & & & \\
\hline
\end{tabular}


chemical, dairy, food, beverage industries and in fermentation and biotechnology [94]. Membrane technology has been used recently in biodiesel purification due to its advantage as an environmental friendly solution in which usage of water in water washing step is avoided [95,96]. Table 3 lists membranes and filters used for glycerol purification.

\subsection{Application of membrane separation technology in glycerol refining}

The application of membrane separation technology in purification of glycerol developed by EET Corporation is depicted in Fig. 4. The patented technology of High Efficiency Electro-Pressure Membrane $\left(\right.$ HEEPM $\left.^{\mathrm{TM}}\right)$ employing an integrated approach in which the membrane units operated in integrated configurations with nanofiltration and/or reverse osmosis units, in a single synergistic unit operation [56].

Mah et al. investigated the fouling pattern of oleic acid and palm oil from glycerol solutions through the ultrafiltration membrane process [97]. A commercial GE PVDF $30 \mathrm{kDa}$ membrane was used in the study to the effect of different palm oil, oleic acid, oleic acid and palm oil concentrations, $\mathrm{pH}$ on membrane flux and rejection ability. It was found from the investigation that the membrane is capable to remove palm oil and oleic acid from glycerol solution with sustainable flux. Jeromin et al. proposed an application of pressure driven membrane technology such as ultrafiltration based on a patent to remove unreacted oil or fat in glycerol-rich solution [98]. The glycerol-rich solution or sweet water from hydrolysis reaction contains a mixture of glycerol, water and impurities such as free fatty acid, unreacted mono-, di-, and triglycerides, inorganic salts and variety of matter organic non-glycerol (MONG). However, frequent cleaning is required due to the fouling of fatty acid. Indok et al. studied the ability of two ultrafiltration polymeric membranes to clarify synthetic glycerinrich solutions containing triglycerides [99]. The membranes tested in the experiment were made of poly (ether sulfone) (PES) and poly (vinylidene fluoride) (PVDF). The reported molecular-weight cut off (MWCO) values were 25,000 and 30,000 Da, respectively. They studied the effects of membrane surface chemistry and solution $\mathrm{pH}$ on the permeation flux and triglycerides retention rate. It was found that in glycerol-water mixtures containing triglycerides, the contribution of the solute to fouling was more severe than that of fatty acid with lower permeation rates being shown. Based on the findings, PVDF membranes were found to provide higher fluxes and lower triglycerides rejection rates (81\%) than PES membranes (91\%). Lazarova tested different types of membrane to purify pre-treated crude glycerol derived from biodiesel production [100]. The biodiesel production uses rapeseed oil as the feed of transesterification process with methanol and $\mathrm{KOH}$ as catalyst. The crude glycerol was pre-treated prior to the membrane separation process. Sulfuric acid was added which resulted in three-phase mixture consisted of a solid salt phase, liquid glycerol phase (middle layer) and liquid fatty acid phase. The liquid glycerol phase was then further treated using pressure driven membrane separation at various transmembrane pressures and feed flow rate. A membrane test unit (Amafilter) for flat membranes was used to compare permeate fluxes and retention capacities of the membranes and purification effect was evaluated based on the data from a UV/VIS analysis. Ismail et al. used a syringe filter $0.45 \mu \mathrm{m}$ as part of pre-treatment process to filter inorganic salts as a result of the neutralization with $85 \% \mathrm{v} / \mathrm{v}$ phosphoric acid [84]. The crude glycerol was then further treated with the ion exchange resin method. An application of nanofiltration polymer membrane as proposed by Traving et al. was able to obtain salt free permeate stream after purification of crude glycerol [101]. The nanofiltration used has pore size between
$0.01 \mu \mathrm{m}$ and $0.05 \mu \mathrm{m}$. Another application of membrane to purify crude glycerol obtained from the transesterification process proposes a series of two or more nanofiltration or reverse osmosis membrane [102]. The process requires at least one of the membranes to have contact angle between $44^{\circ}$ and $56^{\circ}$. A result of glycerol purity of $99.0 \%$ is achievable, filtering and purifying crude glycerol of impurities and salts.

\subsection{Prospect of glycerol refining using membrane technology}

At present, an emerging technology in biodiesel derived crude glycerol purification and refining, that is membrane technology is identified as a feasible solution for the future sustainability of biodiesel production. Membrane technology is considered as an emerging technology that provides much cheaper and decent performance as alternatives for the glycerol purification process $[56,103]$. Although conventional methods, such as combinations of chemical and physical treatments (vacuum distillation, evaporation), produce good quality of purified glycerol, maintaining the facilities, equipment and operational costs are the major hurdles for optimization and cost control. The cost limitations are easily minimized and reduced with the implementation of membrane technology as it requires less energy.

Membrane technology is considered a good alternative with regards to current methods for glycerol purification process. The broad range of membranes applications and advantages has attracted researchers to develop crude glycerol purification methods using membranes. Numerous studies have been engaged in developing the glycerol purification technology based on membrane and other alternatives $[97,99,100]$. However, the incorporation of membrane technology for industrial practice is still found to be lacking despite the obvious advantages of low energy and cost. Perhaps it is largely attributed to membrane disadvantages which have prevented even wider applications of membranebased processes and have not been studied extensively, such as the fouling of the membranes, the durability of membranes and the availability of suitable membranes for specific operations [104]. More research is required to make membrane technology a feasible solution for the sustainability of biodiesel production.

\subsection{Hybrid membrane}

Another exciting prospect of membrane separation technology is the utilization of hybrid membrane. Improvement of membrane performance while combining organic and inorganic materials has been explored in the last few years. The combinations of inorganic/organic material of desired properties have led to a better performance in terms of mechanical and thermal properties from the inorganic part whilst maintaining the flexibility of the organic part of the membranes. According to Zulfikar et al. [105] it was found that water permeability increases with silica content for Poly(methyl methacrylate) (PMMA)- $\mathrm{SiO}_{2}$ composite membranes characterization in which additional amount of tetraethoxysilane were added to PMMA solutions during the membrane formulation. The deposition tetraethoxysilane were made possible due to formation of hydrogen bond between organic phase and inorganic phase during the sol-gel process [106]. Crude glycerol purification process using membrane would be benefited from the improvement of these properties, for example water permeability increase would improve the permeation of water soluble material through the membranes. Improvement in other properties such as thermal and mechanical would produce a membrane with much higher quality, both in terms of performance and sustainability.

\section{Link to Full-Text Articles:}

\section{http://www.sciencedirect.com/science/article/pii/S1364032114009162}

